\title{
A Review on Estimation for Medical Image Registration
}

\author{
Feizhou Peng \\ School of Software Technology, \\ Dalian University of Technology \\ Dalian, China \\ 740919074@qq.com \\ Yanyi Chen \\ School of Software Technology, \\ Dalian University of Technology \\ Dalian, China \\ 479388505@qq.com
}

\author{
Xing Chen \\ School of Optoelectronics Engineering, \\ Nanjing, China \\ cx372747119@163.com \\ Jiajun Xin \\ School of Software Technology, \\ Dalian University of Technology \\ Dalian, China \\ brian1992@126.com
} Nanjing University of Posts and Telecommunications

\begin{abstract}
Medical image registration is very important in many areas and some methods for image registration have been proposed in these decades. However, it is still a serious challenging to estimate the results of medical image registration, because there have been not a golden estimation criterion presented till now. This paper first argues the main content of medical image registration, and then gives a study on existing evaluation methods for medical image registration, including the advantages and shortcomings of each estimation method. At last, it discusses some existing challenges in this area.
\end{abstract}

Keywords- medical imag;image registration; registration estimation

\section{INTRODUCTION}

Medical image registration is extremely important in the medical field $[1,2]$. For example, it is important to help doctors with diagnosis, combining information from multiple imaging modalities into a single image. In addition, it is helpful to study disease progression, monitoring changes in size, shape, position or image intensity over time. What's more important, medical image registration can help a surgeon with image guided surgery or radiotherapy by relating pre-operative images and surgical plans to physical reality of the patient.

The image registration [3] is a kind of method described as an optimization process minimizing the difference between the base image and the input image. The base image is the image against which you compare the image to be registered and the input image refers the image that you wish to register. In the past several decades, many methods for medical image registration have been proposed. The key problem of medical image registration is to find the transformation function.

The paper first introduce the current methods for medical image registration, and in the part 3 , the results of different algorithms are estimated depending on different evaluation criterion. The part 4 pays more attention on the parameters for estimation. The conclusion is given out in the last part.

\section{METHODS FOR MEDICAL IMAGE REGISTRATION}

In the past several decades $[4,5,6]$, many methods for medical image registration have been proposed, falling into three main categories: the point-based algorithms, surfacebased algorithms, volume-based algorithms and information theory-based algorithms. In general, point-based registration algorithms may result in inaccuracies and inconsistencies of image registration because of the low resolution along the longitudinal axis, the small number of corresponding markers, and inaccuracies in their placement or identification. Surface-based registration algorithms depend on a reliable and accurate surface segmentation, which is difficult to achieve in an easy and real-time way. However, volume-based registration algorithms involve the optimization of some similarity measures calculated directly from the voxel values [7]. Information theory based image registration is considered as to maximize the amount of shared information in two images, reducing the amount of information in the combined image.

\section{THE ESTIMATION BY REGISTRATION RESULT}

The earliest estimation methods such as Stochastic and Deterministic Sign Change Criteria were proposed by Early, E. Walter [8]. The nonparametric approach was demonstrate -ed to outperform the conventional image registration criteria for robust registration. Since then, many new approaches are proposed while some new methods on estimating the results of matching come out.

The therapists [9] give estimation methods to evaluate the results of medical image registration, called the subjecttive methods. Both original and matched medical images are presented separately to several therapists. It is testified that the discrimination could achieve to one pixel by eye-estimating [10]. The grades of results are divided four levels, good, accepted, not accepted and bad. The first level is good enough and the error is less than one pixel distance. The second level can be accepted but, the error is bigger than one pixel. The third level is not good enough to accept and the worst level is bad. The error is bigger than 
three pixels. In contrast, the objective method is easy to operate, but the standard is objective and can not be quantified.

Mutual information (MI) $[11,12]$ is currently a popular registration statistics method to scale the similarities between two image sets and for convenience of calculation and analysis. From the abundant literature, it is clear that MI lives up to its reputation of being a general applicable measure and it can be used without any preprocessing, user initialization or parameter tuning. However, from the conclusions of certain comparison studies and from the interest in adaptations of the measure, it can be inferred that MI may not be a universal effective measure for all registration situations. An obvious drawback of mutual information is that the dependence of the gray values of neighbor pixels is ignored. Such situations arise when the images are of low resolution, when the overlapping part of the images is too small or as a result of interpolation methods. A possible solution to failure of MI can be reduced to spatial information, something that is not contained in the measure. On the other hand, when mono modality registration using MI, failures or poor results is often found in that there are many local maxima in MI measure function, which cause problems with optimizer and lead to misregistration.

Estimation by models [13] is an indirect estimating method. Timothy simulated head by a cylinder full of water, with imaginable marks on proper position. Ge made the nails in skull as identifiers in CT/MRI/PET, and estimated the veracity of algorithm by the distance of markers in the images after matching. Evans [14] simulated PET images by MRI images, and tested the registration results after processing images by rotation, moving, resizing, and adding noises. Visible Human CT is usually used as reference image because of its high definition, clear configuration and clear information of location. The distance between the marking points in the matched image can be shown accurately, and such estimation can be quantified. However, the estimation is still far from the real case, especially when simulating a non-rigid object.

The results indicate that the Demons registration algorithm [15] produces the best registration results with respect to the relative overlap statistic; however, it produces nearly the worst registration results with respect to the inverse consistency statistic. This interesting fact illustrates the need to use multiple evaluation statistics to assess the algorithm performance comprehensively.

\section{THE PARAMETERS FOR ESTIMATION}

Some parameters are of great importance for estimation for medical image registration, including quantity of calculation, error parameter and the degree of matching.

Quantity of calculation refers to the time cost and memory cost. The final purpose of algorithm research is to find a swift and effective method for medical application. Thus, the cost of the algorithms including time cost and the memory cost is a significant parameter for estimation. In paper [16], the time cost is given out when analyzing the effectiveness of the algorithm. At the same time, the paper also gives out the clinical estimation to prove the practicality of the algorithm.

The error includes the angle error and the moving error, while the formation of error contains mean error, maximum error, variance, and error median. In paper [17], mean error, maximum error and variance of moving error of $\mathrm{X}$ and $\mathrm{Y}$ label and angle error are used. Error parameter is calculated by:

$$
E=\sum_{i}\left[A\left(x_{i}, y_{i}\right)-B\left(x_{i}, y_{i}\right)\right]^{2}=\sum_{i} e_{i}^{2}
$$

$A\left(x_{i}, y_{i}\right)$ is the test image, while $B\left(x_{i}, y_{i}\right)$ is the reference image.

Every feature point in test image has several desired points to be matched with in reference image [18]. Also every feature point in reference image has several desired points to be matched with in test image. Select one of the desired points with maximum correlation index. The degree of matching is calculated by the formula below.

$\operatorname{Strength}\left(X_{1}, X_{2}\right)=\sum_{Y_{1} \in N\left(X_{1}\right)} \max \left[\operatorname{Similarity}\left(X_{1}, X_{2}, Y_{1}, Y_{2}\right)\right]+\sum_{Y_{2} \in N\left(X_{2}\right)} \max \left[\operatorname{Similarity}\left(X_{1}, X_{2}, Y_{1}, Y_{2}\right)\right]$

In paper [19], the curves about the matching parameters were firstly given out to estimating and comparing the two algorithms. The sharper the curve is, the easier to find out the optimal matching. Secondly, an experiment testing the affection of grey levels was given by calculating the matching parameters under the condition of both full grey levels and reduced grey levels. The affection is quantified by the mean error between those under the two conditions above. Thirdly, the character of anti-noise of the algorithm is estimated by adding white Gauss noise with zero mean and variances of $0.01,0.05,0.1$ and 0.2 into five groups of image. Then draw out a table of the errors of matching parameters under the four conditions to reflect the anti-noise character of the algorithm. This character is finally estimated by the maximum of error, after calculating the mean of five groups under different conditions. At last, the objective estimation method is also used.

\section{CONCLUSION}

Medical image registration is extremely important in the medical field. Current algorithms for medical image registration include four categories: the point-based algorithms, surface-based algorithms, volume-based algorithms and information theory-based algorithms. However, how to estimate the results of medical image registration is a challenging, because there is not a golden criterion presented. The paper analyzes several current evaluation methods for medical image registration algorithms. At present, there are still several problems existing on estimating methods [20].

A large number of parameters or algorithm design choices, both subtle and obvious, are selected by relying upon experience and good engineering principles, but without direct evaluation.

The different estimating methods used now are effective to some extent, especially when comparing two algorithms of matching. Researchers have not found an excellent method or a standard process to analyze the matching 
algorithms. Parameters and methods have their limitedness and shortcomings.

It is ineluctable to compare one algorithm with another, as more and more new algorithms of medical image matching are proposed. In order to find an ideal algorithm for clinical use, estimation should be paid more and more attention. There is still a long way to find out the "golden standard", which is also our next step.

\section{REFERENCES}

[1] Josien P W Pluim, J B Antoine Maintz, Max A Viergever. Mutual information based registration of medical images: a survey. IEEE $\mathrm{T}$ Med Imaging 2003; 22: 886-1004

[2] [2] Feng Lin, Guan Huijuan, Teng Hongfei. Advances in medical image registration based on mutual information. J Biomed Eng 2005; 22: 1078-1081.

[3] [3] Min Tang, Feng Chen. A Qualitative Meta Analysis Review on Medical Image Registration Evaluation. International Workshop on Information and Electronics Engineering (IWIEE) 2012: 499-503.

[4] [4] Barbara Zitová, Jan Flusser. Image registration methods: a survey. Image and Vision Computing, Volume 21, Issue 11, October 2003. Pages 977-1000.

[5] [5] Dengwang Li, Honglin Wan, Hongjun Wang, Yong Yin. Medical Image Registration Framework Using Multiscale Edge Information. Procedia Engineering, Volume 29, 2012, Pages 2480-2484.

[6] [6] P. Markelj, D. Tomaževič, B. Likar, F. Pernuš. A review of 3D/2D registration methods for image-guided interventions. Medical Image Analysis, Volume 16, Issue 3, April 2012, Pages 642-661.

[7] [7] Yunkai Zhang, James C. H. Chu, Wenchien Hsi, Atif J. Khan, Parthiv S. Mehta, Damian B. Bernard, Ross A. Abrams. Evaluation of four volume-based image registration algorithms. Med Dosim 2009; 34: $317-322$.

[8] [8] E.Walter, L.Pronzato, A.Venot, "Theoretical properties of sign change criteria for robust off-line estimation" Automatic, vol.25, pp.949-952, 1989.

[9] [9] QIN Bin-jie, ZHUANG Tian-ge, "Multi-resolution registration of MR and CT images based on correlation ratio similarity measure,"
Chinese Journal of Biomedical Engineering, Vol.22 No.1 February 2003.

[10] [10] Studholme, C, Hill DLG, Hawkes DJ. "Automated 3-D registration of MR and CT images of the head $[\mathrm{J}]$ " Med Image Analysis, 1996, 1 (2): 163-175.

[11] [11] ZONG Ren-he, ZHU Xiang-sheng, LIU Liang-cheng, ZENG Gang-yan. "Mutual Information Based Medical Image Registration" Computer Technology and Application Development 2004.

[12] [12] GAO Zhi-yong, GU Bin, LIN Jiarui, "Implementation of Mutual Information Based Medical Image Registration Methods" Journal of Biomedical Engineering 2003:20(3):476-478

[13] [13] ZHANG Jian-ge "Overview on Medical Image Registration" Academic Journal of Shanghai Second Medical University 02585898(2003)02-0180-03

[14] [14] Evans AC, Collins DL, Nellin P, et al. "Correlative analysis of three imensional brain images computer- integrates surgery [J]" Technology and, Clinical Applications, 1996, 99-144.

[15] [15] Brian B. Avants, Nicholas J. Tustison, Gang Song, Philip A. Cook, Arno Klein, James C. Gee. A Reproducible evaluation of ANTs similarity metric performance in brain image registration. Neuoimage 2011; 54: 2033-3044.

[16] [16] HAI Miao, SHEN Yi, "Research on automatic registration method for 3D multi-modal images" Chin Med Imaging Technol, 2005, Vol.21, No.5

[17] [17] PENG Jing-lin, ZHANG Jing, LI Shu-tao, WANG Lian-hong, "Medical Image Registration Based on Expanded PV Interpolation and Hybrid Optimization Algorithm", ACTA ELECTRONIC SINICA Vol.34, No.5 May, 0372-2112(2006) 05-0962-04

[18] [18] ZHAO Yong-ming, ZHANG Su, ZHANG Zhao, CHEN Ya-zhu, "A Non-rigid Medical Image Registration Algorithm Based on Modally Controlled Free Form Deformation", Space Medicine \& Medical Engineering, 240-245, Vol. 18, No.4, Aug.2005

[19] [19] LI Ke, LIU Chang-chun, LI Tong-lei, "Medical registration approach using improved maximization of mutual information", Journal of Shandong University (Engineering Science), Vol.36, No.2, Apr. 2006, 1672-3961(2006)02-0107-04

[20] [20] Ximiao Cao, Qiuqi Ruan. A survey on evaluation methods for medical image registration. In: 2007 IEEE/ICME International Conference on Complex Medical Engineering, 2007, p. 718-721. 